\title{
Emploi de la stratégie de la formation à distance pour développer quelques compétences linguistiques et communicatives des enseignants non spécialisés de FLE
}

\section{Tag Al Din Mohammed Eid}

Maître de conférences de la méthodologie de FLE à la faculté de pédagogie Université de Minia

\section{Résumé en français}

omme les futurs enseignants non spécialisés souffrent
$d u$ manque des compétences linguistiques et
communicatives de FLE. Par conséquent, le chercheur a essayé dans la recherche actuelle de développer un certain nombre de ces compétences chez eux. Pour préciser la problématique de la recherche, il a appliqué un questionnaire des besoins de formation aux futurs enseignants non spécialisés de FLE inscrits à l'unité des études supérieures à faculté de pédagogie de Minia en 2018 pour avoir un diplôme général en pédagogie. , il a profité de l'analyse de ce questionnaire à élaborer une grille des compétences linguistiques et communicatives utiles destinée aux futurs enseignants. Il a désigné un stage qui compte trois unités ayant un contenu intéressant $d u$ vocabulaire, de règles grammaticales et des activités de communication orale en français. Grâce au progrès des outils technologiques le contenu de ce stage était à la disposition des étudiants inscrits au diplôme général à faculté de pédagogie de Minia au deuxième semestre en 2018 d'après de CD d'après l'internet.Ils ont appris le stage à distance. Pour mesurer l'efficacité de ce stage basé sur la formation à distance, le chercheur a appliqué les pré/post tests de vocabulaire, de grammaire et de communication orale. En analysant statistiquement les résultats des membres de l'échantillon par le programme de SPSS, le chercheur a constaté que l'apprentissage de stage en fonctionnant la formation à distance a un effet très positif à améliorer les compétences linguistiques et communicatives chez les futurs enseignants non spécialisés.

\section{Introduction}

La formation initiale des enseignants de FLE en Egypte se fait exclusivement aux facultés de pédagogie selon un système 
intégral. En effet, ces facultés présentent deux types de formation: une formation académique et une formation pédagogique. Les départements de la langue française soit aux facultés de langues soit aux facultés de lettres qui sont responsables de la formation académique ne présentent pas de formation pédagogique alors qu'ils mettent l'accent sur les connaissances académiques approfondies de la langue française. D'ailleurs, ces connaissances se présentent d'une manière monotone et non applicable. Après l'obtention de la licence, beaucoup de diplômés non pédagogues ne sont pas prêts de faire les cours en classes de FLE et ils souffrent du manque des compétences linguistiques, communicatives et professionnelles nécessaires aux enseignants de FLE. Ainsi, ce problème paraît dans la plupart des pays francophones. Dans ce contexte, Ebtehal Abdel Moneim (2012) a désigné un référentiel en 6 compétences essentielles exigées pour les futurs enseignants de FLE en Egypte dont la compétence linguistique, la communication, sont venues en premier(5). De la même façon, un certain nombre de chercheurs vietnamiens ont organisé un séminaire doctoral en 2012 afin de développer les compétences professionnelles de futurs enseignants de FLE, ils ont appliqué un questionnaire aux étudiants de la dernière année universitaire au sein des départements de français. Les résultats ont montré que la plupart des étudiants s'intéressaient aux connaissances disciplinaires et la majeure partie des étudiants exprime des faiblesses dans la mise en œuvre des techniques de classe.(6)

Un des buts principaux de l'enseignement-apprentissage de français, est:apprendre à se comporter de manière adéquate dans des situations de communication où l'utilisateur aura quelque chance de se trouver en utilisant les codes de la langue cible. Au début de XXI siècle, cet enseignement-apprentissage s'articule autour du développement des compétences générales et communicatives langagières de l'utilisateur/apprenant surtout la parution du Cadre européen commun de référence pour les langues ( CECRL) en 2001. 
Le monde de l'enseignement a emprunté la notion de compétence en éducation au domaine de la formation professionnelle, pour désigner initialement les personnes bien qualifiées. Progressivement, cette notion arrive à être utilisée comme considération relative à un acte à pratiquer, à une tâche à effectuer, à un problème à résoudre. (20)

Dans le domaine éducatif, Olivier Reboul (1981) propose que la fin de l'enseignement ne soit de donner, seulement des informations, des savoir-faire, ou même des savoirs purs, mais avoir une compétence, et il ajoute que l'enseignement ne se fait sans enseignants jouissant d'une compétence reconnue dans leur matière.(10:57-61) En ce qui concerne l'apprentissage des langues étrangères les compétences linguistiques communicatives avec toutes les composantes fait partie des compétences principales requises pour l'exercice du métier de l'enseignement. Comme les langues étrangères sont aujourd'hui enseignées à des fins communicatives, contrairement à auparavant où l'on enseignait les langues étrangères à des fins culturelles, il est demandé à l'enseignant d'être lui-même un bon communicateur doté nécessairement d'une compétence communicative. Dans ce contexte Salih Bachir (1984) indique que le futur enseignant de FLE doit acquérir un certain nombre de compétences: $(12: 24)$

1. Une compétence linguistique et une compétence de communication.

2. Aptitude à enseigner une langue étrangère : connaissance de la linguistique en tant que science et de la didactique des langues étrangères ;

3. Une maîtrise des méthodes de langue à enseigner ;

4. Une compétence en culture générale.

Les futurs enseignants de FLE formés dans les facultés de pédagogie ont l'occasion d'acquérir ces compétences pendant leur formation initiale dans les salles des cours, dans les laboratoires de langue ou dans les écoles où ils suivent des cours pratiques. La formation initiale est conçue comme une 
préparation permettant au futur enseignant de s'adapter à tout type de situation d'enseignement. De plus, la formation initiale doit permettre une bonne maîtrise de la langue cible. Soulignons que certains programmes actuels proposés par les facultés de lettres et les facultés des langues ne sont pas pertinents à former les futurs enseignants non spécialisés de FLE, donc, la formation initiale est incomplète.

De cette façon, le besoin de la formation continue reste nécessaire. ; la formation continue succédant logiquement à une formation initiale, devrait avoir pour but premier de perfectionner les compétences des sujets. La formation continue peut être faite traditionnellement dans les salles des cours comme les centres des langues et le ministère de l'enseignement le fait. Grâce à l'énorme développement de la technologie de la communication, il est devenu très facile de faire la formation des enseignants en ligne. La formation en ligne donne au futur enseignant l'occasion de recevoir de grandes quantités de connaissances chez lui n'importe quand et n'importe où. Tant la recherche actuelle s'intéresse à la formation à distance, on ne s'agit pas la formation traditionnelle qui se fait en papiers, mais on s'agit la formation électronique par l'ordinateur. On peut exécuter ce type formation à distance plus facilement à la formation continue qu'à la formation initiale, et d'une manière individuelle que collective. $(27: 11)$

En effet, la formation à distance électronique a été employée pour présenter des connaissances de différentes disciplines : mathématiques, sciences langues etc. En parlant de la formation des enseignants de FLE on doit indiquer que l'Agence universitaire de la Francophonie (AUF), a présenté un certain nombre de modules de formation en ligne. Cette offre s'adressait aux enseignants et enseignants-chercheurs des départements de français, et aux centres de langues.(35) En vue de la pertinence de la stratégie de formation à distance en ligne, le chercheur a essayé dans la recherche actuelle de l'employer à élaborer le contenu d'une formation destinée aux futurs enseignants non spécialisés qui compte trois unités afin de 
développer les compétences de lexique, de grammaire et de la communication chez eux.

En travaillant sur la formation des enseignants, Aniceto Mapfala (2016) indique que nous devons nous préoccuper d'abord d'analyser nos propres pratiques de formation, ensuite d'avoir un regard des futurs enseignants par rapport à leur formation et à la question de l'enseignement des languescultures et nous devons enfin mieux comprendre si l'origine culturelle et le plurilinguisme jouent un rôle primordial dans leur formation.(2-12) D'où la recherche actuelle vise à développer des compétences générales de futurs enseignants non spécialisés de FLE. Parmi ces compétences, le développement des savoirs et des savoir-faire interculturels qui se trouvent au cœur des préoccupations didactiques.

\section{Problématique de la recherche}

Beaucoup de compétences linguistiques et professionnelles de l'enseignant de FLE sont faibles chez les diplômés non pédagogues des départements de français aux facultés de lettres et de langues. Le chercheur a touché à cette vérité d'après son travail comme maître de conférences de la méthodologie de FLE à la faculté de pédagogie au cours de l'interview faite avec un groupe d'entre eux qui voudraient s'inscrire au diplôme général à la faculté de pédagogie. Pendant cette interview, il leur a demandé de se présenter et de citer les raisons de l'inscription à ce type d'étude. Beaucoup d'étudiants ont trouvé de difficultés d'une part à comprendre les questions qu'il leur a posées ; d'autre part, ils ne pouvaient pas exprimer oralement leurs idées en français.

En faisant les cours du contenu de Curricula aux mêmes étudiants au deuxième semestre en 2018, le chercheur a demandé à un certain nombre d'entre eux de jouer le rôle de l'enseignant de FLE et d'après l'observation de leur présentation, il a découvert qu'ils souffraient du manque de compétences professionnelles et linguistiques de l'enseignant de FLE. Ils avaient peut-être des connaissances académiques théoriques 
mais, ils ne pouvaient pas les employer en classe de langue quand même. Pour vérifier ce problème d'une manière plus logique, on a appliqué un questionnaire (Annexe 1) des compétences nécessaires aux enseignants de FLE. Ce questionnaire compte six composantes concernant les compétences linguistiques, les compétences communicatives, les compétences du processus de l'enseignement, les compétences de gestion de classe, les compétences de l'évaluation et les compétences de professionnalité. D'après l'analyse des résultats de ce questionnaire, on a constaté que les futurs enseignants non spécialisés ont affirmé l'utilité de toutes les compétences mais, ils ont donné un grand soin aux compétences linguistiques, et aux compétences communicatives. En justifiant leur préférence de suivre des cours de didactiques de FLE qui leur donnent la possibilité d'acquérir les quatre autres compétences pendant leurs études au diplôme général c'est pourquoi, le chercheur a décidé de faire recherche actuelle qui vise à présenter un stage basé sur la formation à distance en ligne destinée aux futurs enseignants non spécialisés afin d'améliorer les compétences linguistiques et communicatives chez eux. En effet, le contenu de ce stage est varié, et il est présenté par la stratégie de la formation à distance pour donner aux futurs enseignants de travailler individuellement chez eux.

\section{Donc, la question principale de cette recherche est :}

Dans quelle mesure le stage basé sur la formation à distance participe à développer les compétences linguistiques et communicatives des futurs enseignants non spécialisés de FLE ?

Cette question centrale peut être déclinée de la façon suivante pour mieux la préciser :

1. Sous quelle forme et de quelle façon peut-on formuler le contenu du stage destiné aux futurs enseignants non pédagogues de FLE ?

2. Quelles sont les compétences linguistiques que ce stage vise à développer? 
3. Quelles sont les compétences communicatives que ce stage vise à développer?

\section{Limites de la recherche}

1. La recherche a été appliquée seulement sur les futurs enseignants non spécialisés de FLE, inscrits au diplôme général à la faculté de pédagogie de Minia en 2018.

2. Le contenu du stage est basé sur la stratégie de la formation à distance électroniquement soit en ligne soit enregistré sur des disquettes.

3. Ce stage compte trois unités : la première est consacrée à l'apprentissage du vocabulaire, la deuxième présente un certain nombre des règles grammaticales françaises et la troisième présente des règles de la phonétique française et des fiches pédagogiques de communication orale.

4. Le contenu du stage est fortement lié au contenu de livre club@dos plus le manuel de FLE que les futurs enseignants doivent enseigner aux étudiants de cycle secondaire en Egypte.

\section{Terminologie de la recherche}

\section{Formation}

Le dictionnaire français Larousse définit la formation comme un développement et modification de l'organisme qui rend l'individu capable d'exercer les fonctions de reproduction.(28)

Le nouveau Petit Robert définit la formation comme : " l'éducation intellectuelle et morale d'un être humain » 25 : 1077).

\section{Formation professionnelle}

L'encyclopédie Larousse définit la formation professionnelle comme un ensemble des mesures permettant la formation à un premier emploi, l'adaptation ou la conversion à un nouvel emploi, la promotion ou encore l'acquisition, l'entretien ou le perfectionnement des connaissances et qui sont prises en charge par l'État et l'employeur.(29) 


\section{Compétence}

Dans le Nouveau Petit Robert (2011), la compétence désigne : "Savoir implicite grammatical et lexical intégré par l'usager d'une langue naturelle et qui lui permet de former et de comprendre dans cette langue un nombre indéfini de phrases jamais entendues » (25: 486). Selon Mounin (1974), la compétence est une " notion fondamentale qui désigne la connaissance implicite qu'un sujet a de sa langue ».(24) Legendre (2005) précise que la compétence est une " habileté acquise grâce à l'assimilation de connaissances pertinentes et à l'expérience et qui consiste à construire et à résoudre des problèmes spécifiques » (22. 250).

\section{Procédure de la recherche}

\section{Pour atteindre l'objectif de cette recherche, le chercheur a suivi les étapes suivantes:}

1. Chercher des informations pertinentes concernant le sujet de la recherche dans les périodiques, dans les revues, dans les livres et sur l'internet.

2. Formuler le contenu de la formation destinée aux futurs enseignants non spécialisés qui compte trois unités, enregistrées sur des disquettes.

3. Elaborer une grille de quelques compétences linguistiques et communicatives des futurs enseignants non spécialisés de FLE.

4. Elaborer un pré/post test de vocabulaire.

5. Elaborer un pré/post test de grammaire.

6. Elaborer un pré/post test de communication orale.

7. Choisir l'échantillon de la recherche parmi les étudiants inscrits au diplôme général du département du français à la faculté de pédagogie de Minia.

8. Appliquer les prés tests aux futurs enseignants non spécialisés de FLE.

9. Distribuer les disquettes sur lesquelles le contenu de la formation est enregistré aux futurs enseignants et 
télécharger le même contenu sur leurs pages de Facebook afin qu'ils puissent apprendre ce contenu de la formation à distance chez eux.

10. Appliquer les post-tests aux futurs enseignants de FLE.

11. Faire la statistique et analyser les résultats.

\section{Pertinence de la recherche}

1. Orienter les professeurs aux départements de français aux facultés de pédagogie en Égypte à choisir le contenu de programme de formation convenable aux futurs enseignants.

2. Coopérer avec les responsables du ministère de l'enseignement en Egypte à élaborer une formation destinée aux enseignants de FLE en service afin d'améliorer leurs compétences linguistiques et communicatives.

3. Télécharger le contenu de la formation élaborée par le chercheur sur l'internet en vue de développer les compétences des enseignants de FLE soit en Egypte soit aux pays francophones.

\section{Echantillon de la recherche}

Le chercheur a choisi l'échantillon de la recherche parmi les étudiants inscrits au diplôme général à faculté de pédagogie de Minia en 2018. Ces étudiants sont issus des départements de français aux facultés de lettres et de langues. Cet échantillon compte 60 étudiants sous un seul groupe expérimental. En fait, le chercheur a choisi ces étudiants pour deux raisons:

1. Ils étaient inscrits au diplôme général à la faculté de pédagogie afin d'être futurs enseignants de FLE c.à.d. ils ont besoin d'avoir les compétences linguistiques, communicatives et professionnelles nécessaires à l'enseignant de FLE.

2. Le chercheur faisait des cours de Curricula à ces étudiants au deuxième semestre en 2018 ce qui a facilité sa tâche à appliquer cette recherche. 


\section{Outils de la recherche}

Le chercheur a utilisé les outils suivants pour justifier les résultats de la recherche:

1. Un questionnaire des besoins des futurs enseignants non spécialisés de FLE.

2. Une grille de quelques compétences linguistiques et communicatives des futurs enseignants non spécialisés de FLE.

3. Un pré/post test de vocabulaire.

4. Un pré/post test de grammaire.

5. Un pré/post test de communication orale.

\section{HYPOTHESES DE LA RECHERCHE}

1. Il y a une différence significative entre la moyenne des notes des futurs enseignants non spécialisés de FLE au test du vocabulaire de français avant et après l'application en faveur du post test.

2. Il y a une différence significative entre la moyenne des notes des futurs enseignants non spécialisés de FLE au test de la grammaire de français avant et après l'application en faveur du post test.

3. Il y a une différence significative entre la moyenne des notes des futurs enseignants non spécialisés de FLE au test de la communication orale en français avant et après l'application en faveur du post test.

\section{Buts de la recherche}

Cette recherche vise à:

1. Préciser les compétences linguistiques et communicatives principales des futurs enseignants non spécialisés de FLE.

2. Elaborer le contenu d'un stage basé sur la formation à distance par ordinateur destinée aux futurs enseignants non spécialisés de FLE.

3. Mesurer l'effet de la stratégie de formation à distance par ordinateur sur le développement des compétences linguistiques et communicatives principales des futurs enseignants non spécialisés de FLE. 


\section{Cadre conceptuel}

Cette partie de la recherche traite avec plus de détails les points suivants :

1. Quels sont les types de la formation?

2. Comment peut-on former les enseignants de FLE ?

3. Y a-t-il des différences entre la formation à distance et la formation en ligne?

4. Quelles sont les compétences les plus exigées des enseignants de FLE ?

5. La recherche s'intéresse à quels types des compétences? et pourquoi?

\section{Formation initiale et formation continue, deux mondes de l'enseignement}

Comme son nom l'indique, la formation initiale est destinée à l'étudiant qui souhaite apprendre les bases d'un domaine professionnel, et s'orienter vers une profession. Elle fournit ainsi les compétences et les savoirs nécessaires pour exercer un premier métier. Elle permet ainsi l'obtention d'un diplôme, et sa durée peut varier d'un type de cursus à un autre.

Dans certain pays, comme la Roumanie, la formation des futurs enseignants est une formation " précoce "préuniversitaire" » (16:60) se faisant dans les lycées.

Quant à la formation continue, elle s'adresse à des enseignants en service qui sont déjà pleinement intégrées dans le marché de l'emploi. Il s'agit ainsi de développer ou d'améliorer de nouvelles performances chez eux. Cette formation pourra venir compléter une formation initiale terminée par le passé, ou bien différer complètement de domaine dans le cadre d'une reconversion professionnelle. Ce type de cursus s'adapte directement aux besoins des professionnels et des entreprises. Autrement dit, la formation continue doit être au service des compétences professionnelles, les développer, voire chercher à les faire "mûrir ». La formation continue doit donc continuer tout au long de la vie de l'enseignant. Nous oserons dire que 
quand l'enseignant s'arrête de se former, il s'arrête d'être utile en tant que professeur.

Pour la formation des enseignants de FLE, elle est avant tout une formation continue, venant en complément de la formation initiale. «La formation canonique de cette formation est le stage, » $(4: 193-204)$.

De cette façon, la formation continue poursuit un double but ; d'une part, elle a pour objectif le recyclage qui s'articule autour des changements importants : "réformes de structures, introduction de nouveaux programmes, de nouveaux moyens d'enseignement, de nouvelles technologies... » $(18,1996$ : 29).

\section{Les principales différences entre ces deux modèles de formation}

La première grande différence entre ces deux formations est le public auquel elle s'adresse. En, effet, la formation initiale s'adresse aux étudiants tandis que la formation continue s'adresse à des personnes déjà diplômées qui souhaitent ajouter une corde à leur arc ou monter en compétence.

La seconde différence notable est le prix d'accès à ces formations. En effet si certaines formations initiales peuvent être payantes, la gratuité existe. Cependant en matière de formation continue, tous les cursus sont payants.

Enfin, la troisième et la dernière grande différence entre ces deux typologies de formation reste l'organisation des cours. La formation initiale est un « temps plein » qui pourra cependant être mené en alternance (la partie pratique entre pleinement dans le cadre de la formation dans ce genre de formation initiale), alors que la formation continue, elle est à mener en dehors des heures de travail, et devront ainsi être menées les soirs, weekends, et durant le temps libre. Il sera cependant possible dans certains cas de négocier un aménagement horaire avec l'employeur pour réduire le temps du travail momentanément.(26) 
En ce qui concerne la formation des enseignants de FLE, la formation initiale se fait officiellement aux facultés de pédagogie. Quant aux diplômés non spécialisés issus aux départements de français des autres facultés et qui veulent occuper la profession des enseignants de FLE, ils y reçoivent une formation initiale académique et profonde en français, donc, ils ont besoin d'une formation contenue que met l'accent sur les compétences communicatives et professionnelles des enseignants de FLE.

\section{La formation des enseignants de FLE}

La formation des enseignants de FLE désigne: la préparation à un métier, à une compétence et à l'exercice d'une fonction. Elle se base sur deux volets : le premier est la formation académique et didactique, voire théoriques (savoirs), et le deuxième est la formation professionnelle (le savoir-faire).

Porcher(2004) souligne que la formation des enseignants doit être partiellement linguistique c.à.d. énonciative et pragmatique, exprimée en actes de parole que l'objectif consiste à apprendre à réaliser dans la langue considérée et partiellement elle doit être littéraire concernant le phénomène capital de la manifestation d'une langue. Mais un enseignement s'adresse aussi à l'élève et porte aussi sur une culture. Psychologie, sociologie et anthropologie sont donc nécessaires à la formation de l'enseignant, $(9: 14)$ Suzanne Moftah (2013) indique que l'enseignant doit établir un réseau relationnel avec des partenaires, enseignants et non enseignants. Pour cela, sa formation devra s'ouvrir sur le monde actuel et devra porter sur une approche interdisciplinaire.(13:9)

Nous estimons que former des adultes au métier d'enseignant de FLE, signifie d'abord les former à la didactique du FLE et à la « culture » de la didactique des langues en général et de la didactique du français en particulier. Pour le comprendre, il convient donc de définir et de connaître les caractéristiques particulières de la didactique du FLE qui la distinguent d'autres didactiques disciplinaires. 
La didactique selon Pagès est « un art du dialogue et de la communication ; comme elle s'interroge sur l'origine du savoir et sur sa mise en forme » $(17: 10)$. En d'autres termes, la didactique s'intéresse à la conceptualisation du savoir tout en cherchant à le légitimer.

Or, étant donné que les langues étrangères sont aujourd'hui enseignées à des fins communicatives, contrairement à auparavant où l'on enseignait les langues étrangères à des fins culturelles, il est demandé à l'enseignant d'être lui-même un bon communicateur doté nécessairement d'une compétence communicative. $(17: 6-17)$

\section{Ainsi, la formation des enseignants à la didactique du FLE devrait :}

-Assurer une bonne maîtrise de la langue enseignée à l'oral comme à l'écrit ;

1. Assurer une connaissance théorique en didactique du FLE et dans les disciplines de référence ;

2. Assurer une connaissance des conditions d'enseignement aux différents niveaux (apprenant, programme, supports, équipement, politique institutionnelle...).

\section{Différence entre formation en ligne et formation à distance}

Il y a une confusion entre les types de formation lorsqu'on parle d'un cours ou d'une formation qu'on ne suit pas de manière présentielle : e-learning, formation en ligne, formation à distance, on essaiera d'omettre cette confusion en présentant la définition de chaque type.

\section{Formation à distance}

La formation dite "à distance » ne peut pas être faite obligatoirement en ligne, et c'est cela la différence importante entre formation à distance et E-learning. Une formation à distance peut être faite par courrier (envoi des cours) par mail, par téléphone, etc. Ce type de formation symbolise l'éloignement géographique entre l'apprenant et le professeur ou cours. L'enseignement à distance se passe de manière non 
synchronisée, les étudiants peuvent obtenir les cours au moment où ils le désirent, la formation à distance désignent une formation soit individuelle soit collective. La flexibilité de la formation à distance est un grand atout.

\section{Formation E-learning}

La formation E-learning signifie réaliser une formation, un cours ou un apprentissage à travers une plateforme numérique, via un ordinateur connecté à Internet. E-learning est devenu un terme assez connu internationalement. Les contenus pédagogiques sont mis en ligne, souvent via un module que l'institut, l'école ou l'organisme met en place. L'apprenant peut être n'importe où géographiquement et suivre les cours où qu'il soit. Mais elle comporte également quelques inconvénients comme la solitude, mais si l'apprenant est bien accompagné et suit une discipline de classe régulière, cela ne pose généralement pas de problème.

La formation en ligne a une caractéristique importante: elle comporte des cours en direct avec les enseignants ou l'échange et l'interactivité sont bien effectifs. Ces cours sont enregistrés pour être relus par les élèves à tout moment, constituant ainsi une bibliothèque classifiée de modules pédagogiques. Ces cours directs sont complétés par des cours indirects, préenregistrés, visualisables à tout moment de la semaine. (30)

Comme les technologies d'information de l'enseignement(TICE) sont devenues largement employées dans les cours des langues dans les centres des langues et dans les universités, le chercheur vise à exploiter les TICE à mettre à la disposition des futurs enseignants non spécialisés un stage qu'il a déjà élaboré afin qu'ils apprennent le contenu de ce stage par la stratégie de la formation à distance. Après avoir élaboré le contenu du stage de formation, le chercheur l'a enregistré sur des disquettes et il a distribué aux étudiants, ensuite ;il a envoyé le contenu du stage par E.mail et enfin ,il a téléchargé ce contenu sur les pages de Facebook de chaque membre du public de la recherche de manière qu'ils peuvent l'apprendre individuellement chez eux. 


\section{Compétence}

Pour Talbot (2007), les compétences sont « des connaissances mises en action de manière efficace face à une famille de situations situées dans un contexte » $(21: 35-48)$ Quant à De Ketele (2001), il nous présente une définition plus détaillée de la notion de compétence :« La compétence est la capacité de mobiliser (identifier, combiner et activer) un ensemble de savoirs, de savoir-faire et de savoir-être pour résoudre une famille de situations-problèmes (et non de simples applications) ou, s'il s'agit d'apprentissages langagiers, de produire des actes de communication significatifs » (15. 42). Pierre Barbés indique que la compétence est un concept très riche et diversifié. C'est pourquoi il suggère un certain nombre d'expressions qui la décrivent et qui peuvent en constituer comme un portrait.(19)

1. La compétence est une qualité de l'être humain.

2. Cette qualité est attribuée à la personne jugée apte à exercer dans les faits et de façons adéquates telles fonctions dans un rôle donné.

3. La compétence est le fruit d'une synthèse résultant de l'application d'un jugement pratique à une tâche dans l'exercice d'un rôle, dont celui d'être pleinement humain.

4. La compétence ajoute une qualité d'intégration de ses savoirs et de son savoir connaître, de son faire et de son être, de son savoir-faire et de son savoir-être.

5. La compétence est la possibilité de produire librement, dans le respect des règles sociales, un nombre indéfini de performances imprévisibles mais cohérentes entre elles et adaptées à la situation.

6. La compétence confère un pouvoir, en particulier dans le domaine professionnel.

7. La compétence est observable à partir d'opérations effectuées (comportements et performances) et elle est évaluable.

8. Elle se manifeste sur une longue période, c.-à-d. qu'elle ne va pas sans une certaine stabilité. 
9. La compétence est précise, en rapport avec un champ d'activités particulier et elle est générale, en ce sens qu'elle reste la même pour un ensemble d'actions multiples dans lesquelles elle se manifeste.

10. La compétence manifestée dans la richesse de la connaissance et la perfection du comportement se mesure à l'exigence du rôle et elle constitue un critère important dans l'attribution d'une fonction à l'intérieur d'une profession.

\section{La compétence linguistique}

Du point de vue purement linguistique, Chomsky souligne que la compétence linguistique est " la connaissance que le locuteur-auditeur a de sa langue » (3:13). Allal (2002) indique que la notion de compétence devient objet de débats scientifiques dans le monde éducatif après la célèbre distinction proposée par Chomsky, dans le cadre de la linguistique générative, entre compétence et performance, «entre ce que je sais et ce que je fais, entre les structures ou fonctions mentales qui expliquent l'action du sujet et les comportements observables qui en résultent » $(1: 78)$.

\section{Les compétences communicatives langagières}

La compétence communicative est expliquée par le Dictionnaire de l'éducation Le Gendre comme étant : "Une habileté acquise, grâce à l'assimilation de connaissance pertinentes et à l'expérience, et qui consiste à circonscrire et à résoudre des problèmes spécifique»(23) La notion de compétence a été reprise par Hymes, précisément dans le cadre de la communication. Pour lui, la compétence de communication représente "une contestation et une extension de la notion chomskyenne de compétence». Il soutient que, même s'il existe une compétence linguistique idéale, celle-ci ne signifie pas la maîtrise pratique d'une langue. Maîtriser une langue, c'est posséder la capacité de s'adapter au contexte communicatif, de produire des phrases correctes conformément au cadre social. (7) Le CECRL accorde une importance particulière à la notion de la communication en mettant l'accent sur les compétences dites 
générales, acquises au cours des expériences passées de l'individu/apprenant, des compétences communicatives langagières. De plus, la participation à des évènements de communication a pour conséquence l'accroissement des compétences de l'apprenant à moyen et à long terme, ce qui signifie que ces compétences évoluent au gré des rencontres et des expériences présentes et à venir. Pour les concepteurs du Cadre de Référence pour les Approches (CARAP), l'idée générale que désigne la notion de compétences est qu'elles sont des unités d'une certaine complexité, qui font appel à différentes «ressources», qui relèvent à la fois des savoir-faire, des savoirs et des savoir-être qu'elles mobilisent.(14)

Les savoir-faire sont les connaissances procédurales qui consistent en la capacité de l'apprenant de mettre en œuvre ses démarches, ses techniques, ses opérations mentales et psychomotrices, ainsi que son propre processus.(34) Ces éléments, liés aux données environnementales, situationnelles, culturelles, lui permettent, par la suite, d'actualiser son potentiel dans une situation déterminée. Le savoir-faire évoque non seulement l'opérationnalisation du savoir, mais une opérationnalisation acquise par l'apprenant, ce qui peut se traduire comme une qualité propre, une qualité devenue intrinsèque, une appropriation du savoir(11). Le développement de ces compétences communicatives construit une problématique chez les futurs enseignants et alimente des réflexions dans le monde de l'enseignement et de la formation que ce soit initiale et/ou continue. Pour bien préciser cette notion, S. Moirand propose dans son ouvrage quatre composantes de la compétence communicative à savoir :

- La composante linguistique : concernant la maitrise des procédés lexicaux,grammaticaux et phonétique de la langue pour pouvoir former des énoncés corrects.

- La composante discursive : qui s'intéresse à la connaissance des normes à respecter dans des situations de communication et d'interaction (quand et comment prendre la parole). 
- La composante référentielle : qui donne une importance à la connaissance des objets du monde et de leur réalisation.

- La composante socioculturelle : qui insiste sur les registres de langues utilisés dans une situation de communication déterminée ».(8:57)

Selon le CECRL, afin de réaliser des intentions communicatives, les utilisateurs/apprenants doivent mobiliser les aptitudes générales ci-dessus et les combiner à une compétence communicative de type plus spécifiquement linguistique. Dans ce sens plus étroit, la compétence communicative comprend les composantes suivantes :

\section{compétences linguistiques ;}

\section{compétence sociolinguistique ;}

\section{compétences pragmatiques.}

Les standards identifiés dans le cadre de ce projet peuvent orienter les professeurs des facultés de pédagogie en Égypte pour choisir le contenu de programme de formation qui permet à leurs apprenants d'atteindre les plus hauts niveaux des standards. Par ailleurs, à la lumière des exemples des indicateurs fournis sous chacun des standards déterminés, peuvent être développés des outils d'évaluation de performance des futurs enseignants. Le chercheur a mis l'accent sur les compétences linguistiques et communicatives en élaborant une grille(annexe 2) qui compte 24 de ces types de compétences et pour améliorer ces compétences les futurs enseignants non spécialisé, il a formulé un stage basé sur la stratégie de formation à distance qui se compose de trois unités : la première unité est consacrée à l'apprentissage du vocabulaire, la deuxième s'intéresse aux règles grammaticales françaises et la troisième met l'accent sur l'amélioration de la communication orale en français.

\section{Le cadre pratique}

\section{1-L'élaboration des outils}

Dans cette partie, nous allons traiter les outils que nous avons élaborés afin de répondre aux questions de la recherche et 
de confirmer les hypothèses déjà mises par le chercheur, ces outils sont :

1. Un questionnaire des besoins de formation des futurs enseignants non spécialisés de FLE

2. Une grille des compétences linguistiques et communicatives utiles aux mêmes enseignants ;

3. Un pré/post test du vocabulaire ;

4. Un pré/post test de grammaire ;

5. Un pré/post test de communication orale ;

6. Un stage basé sur la formation à distance destiné aux futurs enseignants non spécialisés.

Nous proposons sur les lignes suivantes avec plus de détails la technique de l'élaboration de chaque outil.

\section{1-Premier outil : Questionnaire sur les besoins de formation des enseignants non spécialisés de FLE.}

En désignant ce questionnaire, le chercheur a bien profité de l'étude de Ebtehal Abdel Moneim (2012) . (4:15). Ce questionnaire se compose de quarante quatre phrases.il est divisé en six composantes, chaque composante compte un certain nombre d'items, les futurs enseignants doivent bien lire les items de chaque composante et indiquer ce qu'ils d'accord ou pas d'accord avec lui. Le questionnaire compte aussi une question ouverte.

\section{La construction de questionnaire}

Le questionnaire se compose de deux parties, Voici les composantes de la première partie

\section{Première composante : Compétence linguistique}

Cette composante compte neuf items qui mesurent les besoins linguistiques des futurs enseignants non spécialisés de FLE.

\section{Deuxième composante : Communication}

Cette composante compte trois phrases qui mettent l'accent sur les compétences communicatives des futurs enseignants non spécialisés de FLE. 


\section{Troisième composante : Processus d'enseignement}

Cette composante compte neuf items qui précisent le rôle de l'enseignant pendant le déroulement de la séance de l'apprentissage de FLE.

\section{Quatrième composante : Gestion de classe}

Cette composante compte cinq items qui visent à préciser le rôle de l'enseignant à diriger le travail en classe.

\section{Cinquième composante : Evaluation}

Cette composante compte quatre items qui déterminent les compétences de l'enseignant à faire le feedback au travail des apprenants.

\section{Sixième composante : Professionnalité}

Cette composante compte dix items qui s'intéressent aux compétences professionnelles de l'enseignant de FLE.

Pour la deuxième partie, il compte une question ouverte. En répondant à cette question, les futurs enseignants non pédagogues de FLE suggèrent autres compétences utiles que l'enseignant de FLE doit avoir.

\section{2-Deuxième outil: Une grille des compétences} linguistiques et communicatives utiles aux futurs enseignants non spécialisés de FLE.

Pour élaborer cette grille, le chercheur a consulté un certain nombre d'études faites par des chercheurs soit égyptiens soit francophones comme l'étude de Ebtehal Abdel Moneim (2012) qui a proposé un référentiel en 6 domaines destiné aux futurs enseignants en Egypte : la compétence linguistique, la communication, le processus d'enseignement, la gestion de la classe, l'évaluation et la professionnalité. On a consulté aussi l'étude de Suzanne Moftah (2013) qui a appliqué deux questionnaires aux étudiants de 4ème année de licence du Département de français de Taëz au Yémen, ces questionnaires visaient à analyser les besoins professionnels des futurs enseignants d'après deux types de questions: questions ouvertes, et questions à choix multiples. On a profité 
dernièrement du séminaire de doctorat effectué par un certain nombre de chercheurs vietnamiens en 2012. Pour préciser les compétences professionnelles utiles aux futurs enseignants au Vietnam, ils ont appliqué un questionnaire qui a mis en évidence des insuffisances entre savoirs théoriques et compétences professionnelles adaptées au contexte ; celles-ci confirment la volonté d'une nouvelle approche dans l'élaboration du programme de formation des enseignants de FLE en contexte vietnamien.

D'après la consultation de ces études, le chercheur a élaboré un questionnaire qui compte six composantes : la compétence linguistique, la communication, le processus d'enseignement, la gestion de la classe, l'évaluation et la professionnalité. Après l'application de questionnaire aux futurs enseignants non spécialisés, le chercheur a analysé des résultats de ce questionnaire qui ont montré que les étudiants ont donné une grande importance aux compétences linguistiques et communicatives ce qui a poussé le chercheur d'élaborer une grille des compétences linguistiques et communicatives qui compte 24 items (Annexe2)

\section{L'importance de la grille de compétences}

Cette grille des compétences peut jouer un rôle important à orienter les professeurs des facultés de pédagogie en Égypte pour choisir le contenu de programme de formation qui permet à leurs apprenants d'atteindre les plus hauts niveaux des standards. Par ailleurs, elle peut jouer un rôle à préciser des outils d'évaluation de performance des futurs enseignants. En effet, l'identification des compétences nécessaires à la formation des futurs enseignants de FLE participe à permettre de juger de la qualité de ces futurs enseignants. En plus, cette grille de compétences sera un outil permettant de déterminer les caractéristiques d'un enseignant compétent.

\section{3-Troisième outil pré/post test du vocabulaire.}

Le chercheur a élaboré ce test (Annexe 3) afin de mesurer l'efficacité du contenu du stage basé sur la formation à distance 
sur le développement des capacités lexicales chez les futurs enseignants non spécialisés. Pour préparer ce test, on a consulté les sites électroniques : français facile ,Bonjour de France et Podcast français facile qui présentent un grand nombre d'exercices lexicaux et langagiers variés et utiles à mesurer la capacité de l'acquisition du vocabulaire.

\section{3-1.La construction du test}

Ce test compte dix exercices gradués qui vont du simple au complexe, d'abord, les étudiants emploient des mots, ensuite ; ils écrivent des phrases et enfin ils rédigent un texte complet.

- Exercices № 1, 2, 3,4 : Les étudiants observent des images et des dessins et ils écrivent au- dessous des images, les noms des articles dessinés.

- Exercices № 5 et 6 : Les étudiants emploient le vocabulaire concernant le sport en citant un certain nombre des sports individuels et de sports collectifs.

- Exercice № 7 : Les étudiants lisent les phrases et devinent le mot auquel la phrase correspond.

- Exercice № 8 : Les étudiants écrivent les lieux auxquels on va pour acheter les choses indiquées.

- Exercice № 9: Les étudiants écrivent des phrases pour exprimer l'activité de chaque profession.

- Exercice № 10 : C'est l'exercice de la dictée, cet exercice se compose de deux parties: pour la première partie, les étudiants écoutent le texte et complètent les blancs. Quant à la deuxième partie, ils écoutent le texte d'un rythme lent puis, ils écrivent la dictée complète.

\section{3-2.Durée du test}

D’après l'application du pré test, le chercheur a calculé la durée convenable du test.

Durée du test $=\frac{\mathrm{Dp}+\mathrm{Dd}}{2}=\underline{70+50}=60$ minutes.

${ }^{*}$ Dp $=$ Durée du premier étudiant. $*$ Dd $=$ Durée du dernier étudiant. 


\section{3-3.La difficulté et de la discrimination des questions}

En utilisant le programme statistique SPSS, on a calculé les indices de la difficulté et de la discrimination de chaque question de test du vocabulaire, les résultats obtenus sont indiqués dans le tableau suivant :

Tableau № 1: Les proportions de difficulté et de la discrimination des questions

\begin{tabular}{|c|c|c|}
\hline Question & Indice de difficulté & Indice de discrimination \\
\hline 1 ère question & .95 & .85 \\
\hline 2ème question & .91 & .79 \\
\hline $3^{\text {ème }}$ question & .90 & .77 \\
\hline 4ème question & .90 & .77 \\
\hline $5^{\text {ème }}$ question & .87 & .71 \\
\hline 6ème question & .87 & .71 \\
\hline 7ème question & .79 & .64 \\
\hline 8ème $_{\text {euestion }}$ & .77 & .61 \\
\hline 9ème question & .71 & .59 \\
\hline 10 ème question & .24 & .21 \\
\hline
\end{tabular}

A partir des proportions de difficulté et de la discrimination des questions citées dans le tableau № 1, on peut constater que le test du vocabulaire destiné aux futurs enseignants de FLE non pédagogues est valable, donc il est applicable.

\section{3-4.La validité du test}

Le chercheur a appliqué le test à un échantillon des futurs enseignants de FLE non spécialisés inscrits au diplôme général à la faculté de pédagogie de Minia puis, on l'a réappliqué au même échantillon après deux semaines; ensuite, on a calculé le coefficient de corrélation entre les notes des étudiants dans les deux passations du test et enfin ,on a calculé la validité du test d'après l'indice de fidélité qui est .91 ce qui signifie que ce test est valable.

La validité du test $=\sqrt{\text { fidélité }}=\$ \overline{\$ 8}=.94$

\section{3-5.La stabilité du test}

D’après le tableau №2, nous observons que la corrélation est significative au niveau de .01, c'est-à-dire la stabilité est élevée. 
Tableau №2

\begin{tabular}{|c|c|c|c|}
\hline & & $\begin{array}{l}\text { Groupe de } \\
\text { témoin (1) }\end{array}$ & $\begin{array}{l}\text { Groupe de } \\
\text { témoin }(2\end{array}$ \\
\hline $\begin{array}{l}\text { Groupe de } \\
\text { témoin (1) }\end{array}$ & $\begin{array}{c}\text { Corrélation } \\
\text { Signification } \\
\text { N }\end{array}$ & $\begin{array}{c}1 \\
60\end{array}$ & $\begin{array}{l}.94 \\
.01 \\
60\end{array}$ \\
\hline $\begin{array}{l}\text { Groupe de } \\
\text { témoin(2) }\end{array}$ & $\begin{array}{c}\text { Corrélation } \\
\text { Signification } \\
\mathbf{N}\end{array}$ & $\begin{array}{l}.94 \\
.01 \\
60\end{array}$ & $\begin{array}{c}1 \\
60\end{array}$ \\
\hline
\end{tabular}

4-Quatrième outil : Un pré/post test de la grammaire.

Ce test (Annexe 4) est désigné afin de mesurer l'effet du contenu du stage élaboré par le chercheur à développer quelques compétences de l'acquisition et l'emploi des règles grammaticales chez les futurs enseignants non spécialisés de FLE. Pour élaborer ce test, on a consulté les sites électroniques de français facile et de français interactif qui comptent beaucoup d'exercices variés et utiles des règles grammaticales françaises

\section{4-1.La construction du test}

Ce test compte sept exercices variés qui visent à mesurer la capacité des étudiants à employer les formes verbales, les pronoms relatifs, les pronoms personnels, la construction des phrases interrogatives et la transformation du singulier au pluriel.

\section{4-2.Durée du test}

D'après l'application du pré test, le chercheur a calculé la durée consacrée au test.

Durée du test $=\frac{\mathrm{Dp}+\mathrm{Dd}}{2}=\frac{100+120}{2}=110$ minutes.

* Dp $=$ Durée du premier étudiant. ${ }^{*}$ Dd $=$ Durée du dernier étudiant.

\section{4-3.La difficulté et de la discrimination des questions}


Le chercheur à utilisé le programme statistique SPSS afin de calculer les indices de la difficulté et de la discrimination de chaque question de test de grammaire, qui a montré les résultats suivants :

Tableau № 5 : Les proportions de difficulté et de la discrimination des questions

\begin{tabular}{|c|c|c|}
\hline Question & Indice de difficulté & Indice de discrimination \\
\hline 1 ère question & .75 & .61 \\
\hline 2 $^{\text {ème }}$ question & .69 & .58 \\
\hline $3^{\text {ème }}$ question & .81 & .71 \\
\hline 4 ème question $^{\text {'̀̀me question }}$ & .79 & .67 \\
\hline $5^{\text {ème }}$ question & .58 & .44 \\
\hline 6 $^{\text {ème }}$ question & .47 & .41 \\
\hline 7ème question & .36 & .31 \\
\hline
\end{tabular}

D'après les résultats indiqués dans le tableau № $\mathbf{5}$ concernant l'indice de difficulté et l'indice de la discrimination de chaque question, on peut constater que ce test de grammaire est valable, et applicable.

\section{4-4.La validité du test}

Le chercheur a appliqué ce test à un échantillon des futurs enseignants de FLE non spécialisés inscrits au diplôme général à la faculté de pédagogie de Minia deux fois successives a montré que l'indice de fidélité qui est .29 ce qui signifie que ce test est valable.

La validité du test $=\sqrt{\text { fidélité }}=\sqrt{.29}=.54$

\section{4-5.La stabilité du test}

D’après les résultats indiqués dans le tableau № 6, on observe que la corrélation est

significative au niveau de .01, c'est-à-dire la stabilité est élevée.

Tableau № 6

\begin{tabular}{|c|c|c|c|}
\hline \multicolumn{2}{|c|}{} & $\begin{array}{c}\text { Groupe de } \\
\text { témoin (1) }\end{array}$ & $\begin{array}{c}\text { Groupe de } \\
\text { témoin(2 }\end{array}$ \\
\hline $\begin{array}{c}\text { Groupe de } \\
\text { témoin (1) }\end{array}$ & $\begin{array}{c}\text { Corrélation } \\
\text { Signification }\end{array}$ & 1 & .54 \\
& $N$ & 60 & .01 \\
\hline $\begin{array}{c}\text { Groupe de } \\
\text { témoin(2) }\end{array}$ & $\begin{array}{c}\text { Corrélation } \\
\text { Signification } \\
\text { N }\end{array}$ & $\begin{array}{cc}.54 \\
.01\end{array}$ & 1 \\
\hline
\end{tabular}




\section{5-Cinquième outil: Un pré/post test de la communication orale.}

On a élaboré ce test (Annexe 5) afin de mesurer l'efficacité du contenu du stage consacré à l'apprentissage de l'expression orale en français en développant quelques compétences de la phonétique et de communication orale chez les futurs enseignants de FLE non spécialisés. Pour élaborer ce test, on a consulté le site électronique de français interactif qui compte des exercices variés et utiles de la phonétique française.

\section{5-1.La construction du test}

Le test compte neuf exercices variés qui vont du simple au complexe :

- Exercices № 1, 2,: mesurent la compétence des étudiants à employer les voyelles et les consonnes.

- Exercices № 3: mesure la compétence des étudiants à employer les lettres nasales.

- Exercice № 4 : mesure la compétence des étudiants à faire la liaison.

- Exercice № 5 : mesure la compétence des étudiants à faire la transcription phonétiques des mots et des phrases.

- Exercice № 6 : mesure la compétence de la lecture à haute voix en considérant le rythme et l'intonation.

-Exercice № 7: mesure la compétence à compléter un dialogue entre un voyageur et un employé à la gare.

- Exercice № 8 : les étudiants composent un dialogue en imaginant ses actions

- Exercice № 9 : Les étudiants indique les étapes suivies pour préparer une recette.

\section{5-2.Durée du test}

D'après l'application du pré test, le chercheur a calculé la durée consacrée au test.

Durée du test $=\frac{\mathrm{Dp}+\mathrm{Dd}}{2}=\frac{80+70}{2}=75$ minutes.

* $\mathrm{Dp}=$ Durée du premier étudiant. ${ }^{*} \mathrm{Dd}=$ Durée du dernier étudiant. 
5-3.La difficulté et de la discrimination des questions

Afin de calculer les indices de la difficulté et de la discrimination de chaque question de test de la communication, on a utilisé le programme statistique SPSS qui a montré les résultats suivants :

Tableau № 3: Les proportions de difficulté et de la discrimination des questions

\begin{tabular}{|c|c|c|}
\hline Question & Indice de difficulté & Indice de discrimination \\
\hline $1^{\text {ère question }}$ & .77 & .62 \\
\hline $2^{\text {ème }}$ question & .79 & .64 \\
\hline $3^{\text {ème }}$ question & .71 & .59 \\
\hline $4^{\text {ème }}$ question & .69 & .57 \\
\hline $5^{\text {ème }}$ question & .58 & .44 \\
\hline $6^{\text {ème }}$ question & .56 & .41 \\
\hline $7^{\text {ème }}$ question & .51 & .39 \\
\hline $8^{\text {ème }}$ question & .37 & .35 \\
\hline $9^{\text {ème }}$ question & .26 & .21 \\
\hline
\end{tabular}

D’après les proportions indiquées dans le tableau № 3, concernant l'indice de difficulté et l'indice de la discrimination de chaque question, nous pouvons constater que le test de la communication destiné aux futurs enseignants de FLE non spécialisés est valable, et applicable.

\section{5-4.La validité du test}

L'application du test à un échantillon des futurs enseignants de FLE non spécialisés inscrits au diplôme général à la faculté de pédagogie de Minia deux fois successives a montré que l'indice de fidélité qui est .5 ce qui signifie que ce test est valable.

La validité du test $=\sqrt{\text { fidélité }}=\sqrt{\$ 0=.71}$

\section{5-5.La stabilité du test}

D’après le tableau № 4, nous observons que la corrélation est significative au niveau de .01, c'est-à-dire la stabilité est élevée. 


\section{Tableau №4}

\begin{tabular}{|l|c|c|c|}
\hline \multicolumn{2}{|c|}{} & $\begin{array}{c}\text { Groupe de } \\
\text { témoin (1) }\end{array}$ & $\begin{array}{c}\text { Groupe de } \\
\text { témoin(2 }\end{array}$ \\
\hline $\begin{array}{c}\text { Groupe de } \\
\text { témoin (1) }\end{array}$ & $\begin{array}{c}\text { Corrélation } \\
\text { Signification }\end{array}$ & 1 & .709 \\
& $N$ & 60 & .01 \\
\hline $\begin{array}{l}\text { Groupe de } \\
\text { témoin(2) }\end{array}$ & $\begin{array}{c}\text { Corrélation } \\
\text { Signification } \\
\text { N }\end{array}$ & .709 & 60 \\
\hline
\end{tabular}

\section{6-Sixième outil : Le stage de la formation}

\section{6-1. Présentation}

Les études que le chercheur a consultées ont élaboré des grilles des compétences professionnelles, linguistiques ou communicatives des enseignants soit en Egypte soit dans un certain nombre des pays francophones comme Vietnam, Mozambique et Yémen. Le chercheur s'est inspiré l'idée de construire un stage de formation à distance destiné aux enseignants non spécialisés de FLE de l'appel à Candidature pour l'accès aux modules de formation en ligne PRO FLE. Cet appel était lancé par l'Agence universitaire de la Francophonie (AUF). Cette offre s'adresse aux enseignants et enseignants-chercheurs des départements de français, et aux centres de langues des établissements membres de l'AUF situés prioritairement dans les pays en développement ou des pays émergents.

Cette formation à distance avec tutorat, développée par le Centre national d'enseignement à distance (CNED) et le Centre international d'études pédagogiques (CIEP), en partenariat avec le Ministère français des affaires étrangères (MAE) et l'Institut français (IF), propose à l'enseignant de français langue étrangère (FLE) bénéficiaire d'acquérir ou de renforcer des compétences professionnelles en réalisant différentes activités en autonomie ou avec l'aide d'un tuteur. Les modules ont notamment pour objectifs de contribuer à améliorer la qualité de l'enseignement / apprentissage du FLE en offrant des outils et un cadre de réflexion conduisant les enseignants à choisir les démarches les plus efficaces pour favoriser l'apprentissage. 
Le stage élaboré comme outil de la recherche actuelle est un peu différent, il vise principalement à améliorer les compétences linguistiques et communicatives des futurs enseignants non spécialisés. En fait le chercheur a mis l'accent sur ces deux types de compétences seulement en élaborant le stage étant donné que les futurs enseignants non spécialisés ont la possibilité de développer les autres compétences didactiques ,professionnelles et pédagogiques en faisant le diplôme générale pour un an à la faculté de pédagogie.

\section{6-2.0bjectifs généraux du stage.}

Après l'apprentissage de ce stage le futur enseignant non spécialisé doit être capable à :

1. Acquérir de nouveau lexique varié et pertinent.

2. Employer correctement le lexique déjà acquis.

3. Prononcer correctement en Français.

4. Employer les règles grammaticales de français.

5. Engager un dialogue avec un francophone.

6. Exprimer librement ses idées et ses pensées.

7. Comprendre en détail un texte oral ou écrit.

8. Communiquer oralement et par écrit en Français.

\section{6-3.Le contenu du stage}

Ce stage compte trois unités, le contenu de ces unités correspond assez que possible au contenu de manuel «club @dos plus» que les enseignants égyptiens de FLE apprennent aux étudiants du cycle secondaire en Egypte. Le chercheur a essayé de faire cette correspondance afin d'améliorer les compétences linguistiques et communicatives chez les futurs enseignants non spécialisés pour qu'ils soient prêts au marché de travail.

La première unité est consacrée à l'acquisition du vocabulaire français.

La deuxième unité s'intéresse à l'apprentissage d'un certain nombre des règles grammaticales de la langue française. 
La troisième unité concerne l'acquisition des compétences de la communication orale en français.

Nous allons présenter en détails le contenu de chaque unité :

\section{6-3.1. Première unité : L'apprentissage du vocabulaire}

Le chercheur a consulté le site électronique: Français Facile en élaborant cette unité (Annexe 6) Ce site présente une grande quantité du vocabulaire varié et utile à l'apprenant de FLE.

\section{6-3.1.1 Objectifs}

1. Développer le capital lexical des apprenants, en réception et en production.

2. Savoir consulter un dictionnaire unilingue ;

3. Permettre aux apprenants d'entrer progressivement dans le fonctionnement de la langue au niveau lexical ;

4. Développer la mémoire lexicale et la mémoire sémantique chez les apprenants ;

5. Acquérir de vocabulaire varié et utile ;

6. Comprendre un texte oral ou écrit ;

7. Employer le vocabulaire acquis dans des situations de communication ;

\section{6-3.1.2 Supports}

$\mathrm{CD}$, ordinateur connecté à l'internet, dictionnaire, papiers, stylo

\section{6-3.1.3 Contenu de l'unité}

Cette unité est entièrement consacrée à l'apprentissage de vocabulaire, qui se ressemble au vocabulaire de manuel de FLE des étudiants de cycle secondaire en Egypte (Club @dos plus) c.à.d. ce contenu est pertinent aux futurs enseignants qui vont enseigner ce manuel aux étudiants plus tard. Cette unité présente le vocabulaire accompagné des images ce qui éveille 
assez que possible la motivation de l'apprentissage chez les étudiants.

L'unité compte 21 modules dont le contenu comme suit :

1. Modules de № 1 à № 16 présentent de vocabulaire varié concernant les animaux, l'alimentation, les pays, les articles, la famille, les fleurs, les fruits.....

2. Module № 17 présente du vocabulaire concernant les sports.

3. Module № 18 propose de vocabulaire concernant les articles de la casse.

4. Module № 19 présente de vocabulaire concernant les adjectifs des couleurs.

5. Module № 20 propose de vocabulaire concernant les métiers.

6. Module № 21 propose de vocabulaire concernant la maison.

7. Module № 22 propose de vocabulaire concernant les vêtements et les accessoires.

En plus chacun de ces modules est suivi d'un exercice qui mesure l'acquisition et l'emploi de vocabulaire.

\section{6-3.1.4 Méthodologie de travail}

L'apprentissage de cette unité est basé sur la formation à distance qui permet aux futurs enseignants non spécialisés d'apprendre le contenu de cette unité individuellement chez eux. Ils apprennent d'abord le vocabulaire de chaque module, ensuite; ils font les activités qui suivent ce module et enfin ils consultent le chercheur d'après l'internet aux problèmes qu'ils enfacent pendant l'apprentissage du contenu. Le rôle de chercheur est comme un tuteur, il reçoit les interrogations et les observations des futurs enseignants d'après l'E. Mail et le Facebook où il présente des interprétations à leurs questions. 


\section{6-4. Deuxième unité : L’apprentissage de la grammaire}

Le chercheur a élaboré cette unité (Annexe7) afin de développer les compétences grammaticales chez les futurs enseignants non spécialisés. Il a essayé de présenter les règles grammaticales figurant dans le livre (club @dos plus), que les étudiants égyptiens du cycle secondaire étudient comme manuel de FLE. Il a aussi élaboré un certain nombre d'activités en consultant le site électronique Français Facile qui présente des activités intéressantes concernant les règles grammaticales françaises.

\section{6-4.1 Objectifs}

1. Employer les déterminants ;

2. Ecrire des phrases simples au présent de l'indicatif;

3. Raconter des avènements au passé ;

4. exprimer des actions au futur ;

5. Respecter l'accord de genre et de nombre;

6. Ecrire correctement un texte ;

7. Utiliser l'impératif à donner des conseils et des ordres ;

8. Employer les pronoms relatifs;

9. Employer correctement les mots d'interrogation ;

10. Employer les pronoms personnels.

\section{6-4.2 Supports}

$\mathrm{CD}$, un ordinateur connecté à l'internet.

\section{6-4.3 Contenu de l'unité}

Cette unité compte 13 modules qui présentent un certain nombre des règles grammaticales françaises d'une manière graduée qui va du simple au complexe. Chaque module est suivi des exercices utiles qui aident les étudiants à appliquer les règles grammaticales apprises.

1. Module № 1 présente les articles indéfinis et définis.

2. Dans le module № 2 les étudiants reconnaissent les adjectifs administratifs et les articles contractés. 
3. Module № 3 s'intéresse à présenter la structure de la phrase verbale.

4. Module № 4 vise à faire les étudiants respecter l'accord de genre et de nombre.

5. Module № 5 présente les formes du pluriel des adjectifs

6. Module № 6 vise à reconnaître la forme du présent de l'indicatif.

7. Module № 7 présente forme du futur simple et futur proche.

8. Dans le module № 8, les étudiants reconnaissent l'emploi de l'impératif

9. Module № 9 présente la structure du passé composé

10. Dans le module № 10 les étudiants reconnaissent l'emploi de l'imparfait.

11. Module № 11 présente l'emploi des mots d'interrogation.

12. Dans le module № 12 les étudiants savent l'emploi des pronoms relatifs.

13. Module № 13 présente l'emploi des pronoms personnels.

\section{6-4.4 Méthodologie de travail}

Le chercheur utilise la méthode inductive à présenter les règles grammaticales, les étudiants apprennent d'abord la règle ensuite ; ils observent les exemples, et enfin ils font les activités qui se trouvent à la fin de chaque module. Ils ont la possibilité de contacter le chercheur pour demander son aide quand ils en ont besoin d'après l'internet.

\section{6-5-Troisième unité : La communication orale en FLE}

Pour élaborer cette unité (Annexe 8) le chercheur a consulté les sites électroniques: Français interactif et Français Facile qui présentent des explications et des activités intéressantes concernant les règles de la phonétique française. Le contenu de cette unité est très proche du contenu de livre club 
@dos plus enseigné par les étudiants du cycle secondaire en Egypte.

\section{6-5.1 Objectifs}

1. Prononcer les lettres de l'alphabet français ;

2. Maîtriser l'accent aigu, grave et circonflexe.

3. Distinguer les voyelles;

4. Différencier les lettres nasales ;

5. Faire la liaison et l'élision ;

6. Lire correctement un texte écrit ;

7. Comprendre un document oral;

8. Engager un dialogue avec un francophone.

9. Développer la production orale chez les apprenants.

\section{6-5.2 Supports}

$\mathrm{CD}$, ordinateur connecté à l'internet,

\section{6-5.3 Contenu de l'unité}

Cette unité s'intéresse à développer les compétences de la communication orale en FLE, dans cette unité, le chercheur présente d'abord un certain nombre de règles de la phonétique française suivies des activités utiles qui aident les apprenants à bien prononcer en français., ensuite;il présente quatre fiches pédagogiques qui traitent la technique de la communication orale en français, enfin ,il présente cinq modèles de dialogues suivis des activités Le chercheur présente aux apprenants des sites électroniques auxquels, ils peuvent connecter pour écouter oralement le contenu de chaque module.

L'unité compte 17 modules dont le contenu comme suit :

Module № 1 présente les lettres de l'alphabet français et donne des exemples accompagnés des images à chaque lettre. Module № 2 présente les accents aigu, grave et circonflexe.

Module № 3 s'intéresse à différencier entre les voyelles et les consonnes.

Module № 4 guide les apprenants à savoir que le e muet est toujours silencieux. 
Module № 5 présente aux étudiants la manière de la prononciation des voyelles nasales et des semi-voyelles.

Module № 6 guide les étudiants à faire la liaison et l'élision.

Module № 7 présente différents types de l'intonation de la phrase.

Module № 8 présente la manière de faire les syllabes des mots et des expressions.

Module № 9 : présente une fiche pédagogique sur un appel téléphonique à un ami absent.

Module № 10 : traite une fiche pédagogique sur un appel à un médecin.

Module № 11 : traite une fiche pédagogique concernant la fiche d'accueil.

Module № 12: traite une fiche pédagogique concernant l'accueil d'un client.

Module № 13 présente un dialogue : chez la famille d'accueil. Module № 14 présente un dialogue : à la boulangerie.

Module № 15 présente un dialogue : à la boucherie.

Module № 16 présente un dialogue : Visite de l'appartement

Module № 17 présente un dialogue : Acheter un vêtement.

Chaque module compte des exercices utiles qui aident les étudiants à appliquer les règles apprises.

\section{6-4.4 Méthodologie de travail}

Les étudiants apprennent le contenu de cette unité individuellement chez eux. Ils commencent à lire les consignes concernant la prononciation, ensuite ; ils observent les modèles et enfin, ils font les activités qui suivent ce module. Quand ils ont besoin de l'aide du chercheur, ils peuvent le consulter d'après l'internet. Le chercheur à son tour reçoit les interrogations des étudiants et y répond.

\section{Expérimentation de la recherche}

Le chercheur a appliqué cette recherche aux futurs enseignants non spécialisés de FLE qui étaient inscrits à l'unité des études supérieures à la faculté de pédagogie de Minia pour avoir un diplôme général en pédagogie à l'année universitaire 
2017- 2018. ,il faisait les cours de Curricula à ces étudiants au deuxième semestre en 2018 ce qui a facilité sa tâche.

Après avoir terminé l'élaboration de la formation qui compte trois unités destinées aux futurs enseignants non pédagogues, le chercheur a enregistré le contenu de cette formation sur $60 \mathrm{CD}$ et pour mettre le contenu de la formation à la disposition des étudiants il a suivi trois mesures :

1. Il a distribué les $\mathrm{CD}$ aux étudiants

2. Il a également envoyé le contenu de la formation aux étudiants par E.Mail.

3. Il a enfin envoyé le contenu d'après des dossiers joints à un message privé sur la page de Facebook de chaque étudiant.

L'application de ce stage a duré six semaines où les étudiants ont appris à distance le contenu de chaque unité de stage en deux semaines.

Le rôle de chercheur était comme un tuteur pendant que les futurs enseignants fassent le stage à distance, il a suivi ces étapes pendant l'application du stage :

1. Le chercheur a appliqué le pré test du vocabulaire aux futurs enseignants.

2. Les futurs enseignants ont appris à distance la première unité du stage consacrée à l'apprentissage du vocabulaire pour deux semaines.

3. Le chercheur a appliqué le post test du vocabulaire.

4. Il appliqué le pré test de la phonétique aux futurs enseignants.

5. Les futurs enseignants à leur tour ont appris la deuxième unité du stage consacrée à apprentissage d'un certain nombre de règles de la phonétique française suivies de cinq dialogues.

6. Le chercheur a appliqué le post test de la phonétique.

7. Il a appliqué le pré test aux futurs enseignants 
8. Il leur a donné l'occasion apprendre à distance la troisième unité du stage qui présente un certain nombre de règles grammaticales françaises.

9. Il a appliqué le post test de grammaire.

\section{Les problèmes de l'expérimentation et le rôle du chercheur à les résoudre.}

Le chercheur a rencontré un certain nombre de problèmes pendant l'application du stage de formation aux futurs enseignants non spécialisés. Voici quelques problèmes principaux :

1. Certains étudiants n'ont pas de réseau d'internet chez eux et par conséquent, ils ne peuvent pas échanger le contact avec lui, le chercheur leur a conseillé d'apprendre le contenu du stage par l'ordinateur en utilisant les CD et il leur a demandé de lui téléphoner sans hésitation quand ils ont besoin de son aide.

2. Pour certains étudiants, ils ont trouvé des difficultés à comprendre certains lexiques, le chercheur leur a demandé de consulter le dictionnaire électronique soit Larousse soit Robert sois dictionnaire électronique de Google sur le site : https://translate.google.com

3. Pour ceux qui ont trouvé de difficulté à assimiler quelques règles grammaticales et quelques règles de phonétique, le chercheur leur a suggéré de consulter les sites électroniques Français Facile et Français Interactif.

\section{Commentaires des futurs enseignants non spécialisés sur le stage.}

$\mathrm{Au}$ dernier cours de semestre et après l'application du stage, le chercheur a géré une discussion avec les futurs enseignants non pédagogues sur la pertinence du stage. Le chercheur leur a remercié et leur a demandé d'exprimer franchement leurs opinions du stage. Ils ont montré qu'ils ont bien profité du stage et des activités présentées à la fin de chaque module, ils étaient motivés par la présentation intéressante de 
contenu du stage. L'apprentissage du stage en employant la stratégie de la formation à distance leur a donné l'occasion de travailler individuellement, de chercher sur l'internet et de consulter le chercheur au temps de nécessité. Les futurs enseignants non pédagogues souhaiteraient que ce stage soit enseigné pendant leur étude à l'université.

\section{Analyse des résultats}

\section{Pour les résultats du questionnaire}

Le questionnaire concernant les besoins de formation des enseignants non spécialisés de FLE se compose de deux parties : la première partie est un type des questions fermées, elle compte 44 phrases qui ont deux alternances (d'accord et pas d'accord) distribuées sur six composantes. Les réponses à ces phrases peuvent être traitées statistiquement d'aune manière quantitative, le chercheur a d'abord compté le pourcentage de chaque phrase ; ensuite, il a compté le pourcentage de chaque composante (Annexe 9). La deuxième partie du questionnaire est un type des questions ouvertes, elle s'interroge sur les besoins de la formation suggérés par les futurs enseignants.

\section{Voici l'analyse des résultats de la première partie :}

\section{La première composante: Les compétences linguistiques}

Cette composante compte dix items : les phrases (de 1 à 10) qui précisent un certain nombre de besoins linguistiques des futurs enseignants non spécialisés de FLE. D'après l'analyse des réactions des étudiants, le chercheur a compté le pourcentage de chaque item qui étaient comme suit: № $1,3,4: 100 \%$; № 2 : $96.7 \%$;№ $5: 95 \%$; № 6 et 10: $91.6 \%$; № 7: $93.3 \%$;№ 8: $90 \%$;

№ 9: $88.3 \%$; ensuite, le chercheur a compté la moyenne des pourcentages de toutes les items de la composante qui était 94.65\%, (tableau №7). Les pourcentages des items de cette composante sont les plus élevés par rapport aux pourcentages des items des autres composantes, cela indique que les compétences linguistiques sont très importantes, ils viennent au 
sommet des priorités des futurs enseignants non spécialisés de FLE.

\section{La deuxième composante: Les compétences communicatives}

Elle compte six items : les phrases (de 11 à 16) qui visent à préciser les besoins communicatives destinées aux futurs enseignants non pédagogues de FLE .En analysant les réponses des étudiants, le chercheur a compté les pourcentages de chaque phrase qui étaient comme suit.

№ $11: 91.6 \%$; № 12 : $86.6 \%$;№ 13 : $83.3 \%$;№ 14 : 85 $\%$;№ 15: $80 \%$;№ 16 est： $78.3 \quad \%$.En observant ces pourcentages et la moyenne totale des pourcentages de la composante $84.13 \%$ : indiqué dans le tableau № 7 , on peut constater que les compétences communicatives viennent à la deuxième place des priorités des futurs enseignants non spécialisés de FLE.

\section{La troisième composante: Les compétences de l'évaluation}

Elle compte quatre items: les phrases (de 31 à 34) qui visent à préciser les compétences de l'évaluation destinées aux futurs enseignants non spécialisés de FLE .En analysant les réponses des étudiants, le chercheur a compté les pourcentages des items de cette composante qui étaient comme suit :

№ $31: 76.7 \%$; № 32 : $71 \%$; № 33 : $70 \%$; № 34 : 38.3 $\%$.En plus la moyenne totale des pourcentages de la composante 61.65\%: indiqué dans le tableau № 7 ,ces indicateurs constatent que les compétences de l'évaluation viennent à la troisième place des priorités des futurs enseignants non spécialisés de FLE.

\section{La quatrième composante : Les compétences de la gestion de classe}

Cette composante compte cinq items: représentés par phrases (de 26 à 30) qui visent à déterminer un certain nombre des compétences de la gestion de classe .D'après l'analyse des 
réponses des étudiants, le chercheur a compté les pourcentages des items de cette composante qui étaient comme suit :

№ 26 et $28: 38.3 \%$; № 27 : $75 \%$; № $29: 73.3 \%$; № $30: 71.6$.Il a aussi compté la moyenne totale des pourcentages de la composante qui était $61.65 \%$ : indiqué dans le tableau № 7 ,En comparant ces pourcentages aux autres pourcentages du questionnaire, le chercheur a constaté que ces compétences viennent à la quatrième place des priorités des futurs enseignants non spécialisés de FLE.

\section{La cinquième composante : Les compétences de la professionnalité}

Cette composante compte dix items : les phrases (de 35 à 44) qui déterminent les besoins professionnels des futurs enseignants non spécialisés de FLE. Le chercheur a compté le pourcentage de chaque item qui étaient comme suit : № 35 et $39: 36.6 \%$; № $36: 71.6 \%$; № $37: 38.3 \%$; № $39: 73.3 \%$; № 40 : $75 \%$;№ 41 et 43: $60 \%$; ;№ 42 et $44: 41.6$,puis, il a compté la moyenne des pourcentages de toutes les items de la composante qui était 53.46\%, (tableau №7). D’après ces pourcentages le chercheur a constaté que les compétences de cette composante occupent la cinquième place chez les futurs enseignants non spécialisés de FLE.

\section{La sixième composante: Les compétences du processus de l'enseignement}

Cette composante s'intéresse aux compétences didactiques, elle compte neuf phrases (de 17 à 25) Les pourcentages des items étaient comme suit : № 17:36.6\% ; № $18: 336 \%$; № 19 et 24: $50 \%$; № 20 et 21: $38.3 \%$; № $22: 40 \%$;№ 23 et 25 : 60 $\%$,puis, il a compté la moyenne des pourcentages de toutes les items de la composante qui était 45\%, (tableau №7). Cela nous guide que les compétences de cette composante viennent à la sixième et dernière place des priorités des futurs enseignants non spécialisés de FLE.

Quant à deuxième partie du questionnaire, elle compte une question ouverte qui demande aux étudiants de citer les besoins 
de formation plus utiles pour eux. Les étudiants ont rédigé un certain nombre des suggestions que le chercheur a tenues en compte en élaborant le stage de formation.

\section{Pertinence du questionnaire} à :

Le chercheur a bien profité des résultats du questionnaire

1. Elaborer une grille des compétences linguistiques et communicatives, le chercheur a mis l'accent sur ces deux types des compétences car, elles sont les plus exigées du public de la recherche selon l'analyse des résultats du questionnaire.

2. Désigner la Formation destinée aux futurs enseignants non spécialisés qui vise à développer les compétences les plus nécessiteuses d'eux

Tableau № 7 : L'ordre des pourcentages des composantes du questionnaire

\begin{tabular}{|c|c|c|}
\hline & Composante & Pourcentage \\
\hline I & Compétences Linguistiques & $\mathbf{9 4 . 6 5 \%}$ \\
\hline II & Competences Communicatives & $\mathbf{8 4 . 1 3 \%}$ \\
\hline III & Competences D'évaluation & $\mathbf{6 1 . 6 5 \%}$ \\
\hline IV & Competences De Gestion De Classe & $59.3 \%$ \\
\hline I & Competences De Professionnalité & $53.46 \%$ \\
\hline VI & Competences Du Processus D'enseignement & $\mathbf{4 5 \%}$ \\
\hline
\end{tabular}

\section{Quant aux résultats des tests}

Le chercheur a appliqué trois tests avant et après l'apprentissage du stage afin de vérifier la validité des hypothèses de la recherche telles que :

1. Il y a une différence significative entre la moyenne des notes des futurs enseignants non spécialisés de FLE au test du vocabulaire de français avant et après l'application en faveur du post test.

2. Il y a une différence significative entre la moyenne des notes des futurs enseignants non pédagogues de FLE au test de la phonétique de français avant et après l'application en faveur du post test. 
3. Il y a une différence significative entre la moyenne des notes des futurs enseignants non pédagogues de FLE au test de la grammaire de français avant et après l'application en faveur du post test.

Pour vérifier la première hypothèse, le chercheur a appliqué un pré/post test qui mesure l'acquisition des compétences lexicales chez les futurs enseignants non pédagogues de FLE. En examinant les notes des membres de l'échantillon avant et après l'apprentissage de l'unité consacrée au vocabulaire (Annexe 10), le chercheur a observé qu'il y a une amélioration aux notes des étudiants au post test du vocabulaire. Le changement des notes des exercices № 1, 2,3,4 en faveur du post test du indiquent que les compétences № 1 et 9 citées dans la grille des compétences se sont développées chez les étudiants, les indices notes des exercices № 5,6,7,8 au post test affirment le développement des compétences № 2 et 21 et le changement des notes des exercices № 9 et 10 correspondent l'amélioration des compétences № 9 et 18.

Pour constater pratiquement ces relations positives, le chercheur a utilisé le programme électronique SPSS pour traiter statistiquement les notes des étudiants qui a montré les résultats indiqué au tableau № 8.

Tableau № 8.

\begin{tabular}{|c|c|c|c|c|c|c|c|}
\hline $\begin{array}{c}\text { Test du } \\
\text { vocabulaire }\end{array}$ & $\mathrm{N}$ & $\mathrm{Ma}$ & $\mathrm{E}$ & $\mathrm{DL}$ & $\mathrm{C}$ & $\mathrm{T}$ & $\mathrm{S}$ \\
\hline Pré test & \multirow{2}{*}{60} & $\mathbf{4 5 . 9 8 3 3}$ & 13.09482 & \multirow{2}{*}{59} & .743 & 13.244 & $\begin{array}{c}\text { au niveau } \\
\text { de 0. 01 }\end{array}$ \\
\cline { 5 - 8 } Post test & & 64.6000 & $\mathbf{1 6 . 1 8 9 7 6}$ & & & &
\end{tabular}

$\mathbf{N}=$ nombre des étudiants.

$\mathbf{M a}=$ moyenne arithmétique.

$\mathbf{E}=$ écart type.

DL= degré de liberté.

C = Corrélation

$\mathbf{T}=$ valeur de $(\mathrm{t})$ test.

$\mathbf{S}=$ significative.

D'après l'observation des valeurs indiquées dans le tableau concernant les résultats de deux tests du vocabulaire, on peut 
montrer que la valeur de moyenne arithmétique des notes des étudiants au pré test est 45.98 alors que celle-ci de post est 64.60 ; la valeur de l'écart type des notes des étudiants avant l'apprentissage de l'unité du vocabulaire est 13.09 tandis que celle-ci après l'apprentissage de cette unité est 16.18,on peut montrer aussi que la valeur de T Test (13.244) est significative au niveau de.01. Ces résultats nous conduisent à affirmer que l'apprentissage de l'unité du vocabulaire basée sur la stratégie de la formation à distance a participé à enrichir le vocabulaire des futurs enseignants non pédagogues et à développer leurs compétences linguistiques. Les étudiants ont acquis de nouveau lexique, ils peuvent écrire correctement des mots et produire des phrases orales et écrite en employant ce vocabulaire.

Pour justifier la deuxième hypothèse, le chercheur a comparé le notes des étudiants au test de la phonétique avant et après l'apprentissage à distance de la deuxième unité du stage visant à développer un certain nombre compétences de l'expression orale

\section{Pour vérifier la deuxième hypothèse, le chercheur a suivi deux étapes :}

La première étape, il a examiné les notes de pré/post test de grammaire (Annexe 10) appliqué aux futurs enseignants non spécialisés, il a observé qu'il y a une augmentation aux notes des étudiants au post test. Ce changement indique que le test est efficace à mesurer le progrès à acquérir les compétences grammaticales .Cela est clair aux indices qui mesurent l'acquisition des compétences № 9,10,20,21,24 citées dans la grille des compétences Annexe 2.

La deuxième manière, le chercheur a utilisé le programme électronique SPSS pour traiter statistiquement les notes des étudiants qui a montré les résultats indiqué au tableau № 9.

Tableau № 9.

\begin{tabular}{|c|c|c|c|c|c|c|c|}
\hline $\begin{array}{c}\text { Test de } \\
\text { grammaire }\end{array}$ & $\mathrm{N}$ & $\mathrm{Ma}$ & $\mathrm{E}$ & $\mathrm{DL}$ & $\mathrm{C}$ & $\mathrm{T}$ & $\mathrm{S}$ \\
\hline Pré test & \multirow{2}{*}{6} & $\mathbf{2 9 . 2 8}$ & $\mathbf{6 . 1 8 9}$ & \multirow{2}{*}{59} & .783 & $\mathbf{1 8 . 7 9 5}$ & $\begin{array}{c}\text { au niveau } \\
\text { de 0.01 }\end{array}$ \\
\cline { 1 - 3 } Post test & & $\mathbf{4 1 . 4 8}$ & $\mathbf{8 . 0 8 1}$ & &
\end{tabular}


En observant les valeurs indiquées dans le tableau concernant les résultats de pré/post test de grammaire, on peut constater que la valeur de moyenne arithmétique des notes des étudiants au pré test est $\mathbf{2 9 . 2 8}$ alors que celle-ci de post est 41.48; la valeur de l'écart type des notes des étudiants avant l'apprentissage de l'unité de grammaire est $\mathbf{6 . 1 8 9}$ tandis que celle-ci après l'apprentissage de cette unité est $\mathbf{8 . 0 8 1}$, la valeur de T Test (18.795) est significative au niveau de.01. Ces résultats affirme que la stratégie de la formation à distance que le chercheur a employé à présenter la deuxième unité a un effet positif à améliorer les compétences grammaticales chez les futurs enseignants non spécialisés de FLE.

Pour justifier la troisième hypothèse, le chercheur a comparé les notes des étudiants au test de la communication orale (Annexe 10) avant et après l'apprentissage à distance de la troisième unité du stage visant à développer un certain nombre de compétences de l'expression orale chez les futurs enseignants non spécialisés, il a observé que les notes du post test sont meilleurs que celles du pré test. Cela indique que les exercices du test sont pertinents à mesurer les compétences de la communication № 3,4,5,6,7,8,11,12,13,14,15,17,19,20,22,23,24 rédigés dans la grille des compétences Annexe 2 .

Le programme statistiques électronique SPSS affirmé les résultats indiqués dans le tableau № 10

Tableau № 10.

\begin{tabular}{|c|c|c|c|c|c|c|c|}
\hline $\begin{array}{c}\text { Test de } \\
\text { communication } \\
\text { orale }\end{array}$ & $\mathrm{N}$ & $\mathrm{Ma}$ & $\mathrm{E}$ & $\mathrm{DL}$ & $\mathrm{C}$ & $\mathrm{T}$ & $\mathrm{S}$ \\
\cline { 1 - 7 } Pré test & \multirow{2}{*}{60} & 26.3333 & 7.57039 & \multirow{2}{*}{50} & .928 & 18.795 & $\begin{array}{c}\text { au } \\
\text { niveau } \\
\text { de } 0.01\end{array}$ \\
\cline { 1 - 7 } Post test & & 36.1500 & 9.85630 & & &
\end{tabular}

Les valeurs indiqués dans le tableau montre que l'apprentissage de la troisième unité en fonctionnant la stratégie de la formation à distance réalisé le but de développer les compétences de la communication orale en FLE chez les futurs enseignants non spécialisés. 


\section{Recommandations et suggestions}

A la lumière des résultats de cette recherche qui affirment la pertinence de la formation distance à l'apprentissage d'un stage en français, le chercheur recommande de poursuivre l'étude dans ce domaine afin d'améliorer d'autres compétences chez les futurs enseignants non spécialisés comme les compétences interculturelles. Ainsi le chercheur suggère que les départements de FLE adaptent la stratégie de l'apprentissage à distance pour présenter un contenu linguistique aux étudiants, il suggère aussi que le ministère d'enseignement télécharge le contenu de ce stage au site électronique officiel du ministère pour être à la disposition des enseignants en service.

\section{Bibliographie}

\section{Livres et Thèses}

ALLAL, Linda (2002). " Acquisition et évaluation des compétences en situation scolaire », in L'énigme de la compétence en éducation, Bruxelles, De Boeck Université, coll. Raisons éducatives, 77-94.

Aniceto Mapfala(2016): La formation des enseignants de Français Langue Etrangère à l'université dans les contextes plurilingues et multiculturels du Mozambique these de doctorat de l'université de lyon.2016

CHOMSKY, Noam (1971). Aspects de la théorie syntaxique, traduction de MILNER, Jean-Claude, Paris, Éditions du seuil, coll. L'ordre philosophique

DABÈNE, Michel (1994). « La formation au métier d'enseignant : l'enfant pauvre de la didactique des langues ", in Vingt ans dans l'évolution de la didactique des langues '1968- 1988', Paris, Hatier/Didier, coll. LAL, 193-204.

Ebtehal Abdel Moneim (2012) :Élaboration d'un référentiel de compétences pour les futurs enseignants de FLE,thèse de doctorat, Faculté des sciences de l'éducation,Université de Montréal,2012.

G. Ziegler, I. Schneider, G. Torresin et A. Simpson(2012) : Compétences professionnelles des futurs enseignants de 
FLE au Vietnam: de la théorie à la pratique,Actes du Séminaire Doctoral International 2012.

HYMES, Dell Hathaway (1991). Vers la compétence de communication, Paris, Hatier/Didier, coll. LAL. Traduction de France Mugler.

Moirand Sophie (1982) : Enseigner à communiquer en langue étrangère, édition hachette, paris, 1982, p : 57.

PORCHER, Louis (2004). L'enseignement des langues étrangères, Paris, Hachette Éducation, coll. F : Pratique pédagogique.

REBOUL, Olivier (1981), " L'éducation politique et l'école 》, L'école et les valeurs. Actes du congrès mondial des sciences de l'éducation, Québec, Serge Fleury Éditeur, p. 5761

Rinetta Kiyitsioglou-Vlachou(2017) : La maîtrise des compétences linguistiques garantit-elle l'opérationnalisation des savoir-faire interculturels ? Le cas des apprenants hellénophones, revue électronique du centre européen d'études slaves, Publiée en ligne le 08 février 2017.

SALIH BACHIR, Abdullah (1984). Formation des enseignants du français langue étrangère au Soudan : présentation critique, mémoire de magistère en Sciences du langage et de la communication linguistique, Institut de Linguistique et de Phonétique, Université d'Alger.

Suzanne Moftah(2013) : De l'analyse des besoins professionnels à l'élaboration d'un dispositif de formation à l'enseignement du FLE, thèse de doctorat, Université de franche-comté.2013.

\section{Périodiques}

Conseil de l'Europe (2001). Le Cadre Européen Commun de Référence pour les Langues : apprendre, enseigner, évaluer, Paris, Didier.

De Ketele, J.-M., \& Gerard, F.-M. (2005). La validation des épreuves d'évaluation selon l'approche par compétences. Mesure et évaluation en éducation, 28(3). 
IUCU, Romita. (2006). " La formation des enseignants : perspectives et tendances dans le système éducatif roumain », in Carrefours de l'éducation, n 22, p. 57-68.

PAGÈS, Alain (1993). «Perspectives actuelles en didactique du français langue maternelle », in Les enjeux de la didactique $\mathrm{du}$ français, L'école des lettres, $\mathrm{n}^{\circ} 9$, Paris, L'école des lettres, 7-16.

PERRENOUD, Philippe (1996). " Formation continue et développement de compétences professionnelles ", in L'Éducateur, n ${ }^{\circ}, 28-33$.

Pierre Barbés(1990) : Perspectives sur la compétence, pédagogie collégiale, Vol 4 n¹septembre 1990

Rinetta Kiyitsioglou-Vlachou(2017) : La maîtrise des compétences linguistiques garantit-elle l'opérationnalisation des savoir-faire interculturels ? Le cas des apprenants hellénophones, revue électronique du centre européen d'études slaves, Publiée en ligne le 08 février 2017.

Talbot, L. (2007). Quelles compétences professionnelles pour les professeurs des écoles. In M. Bru, \& L. Talbot (dir.), Des compétences pour enseigner : entre objets sociaux et objets de recherche (pp. 35-48). Rennes : Presses universitaires de Rennes.

\section{Dictionnaires}

Legendre, R. (2005). Dictionnaire actuel de l'éducation. (3e éd.). Montréal : Guérin.

Legendre, R, Dictionnaire actuel de l'éducation. Montréal, Québec : Guérin.1993

Mounin, G. (1974). Dictionnaire de la linguistique. Paris : Presses universitaires de France.

REY-DEBOVE, Josette et REY, Alain (dir.) (2011). Le Nouveau Petit Robert, Paris, Le Robert 


\section{Sitographie}

http://www.sufco.fr/formation-continue-vs-

formation-initiale.html 3oct 2018

https://fr.wikipedia.org/wiki/Formation \%C3\%A0 dis

tance

https://www.larousse.fr/dictionnaires/francais/forma tion/34643 le 23-7-2018

http://www.larousse.fr/encyclopedie/divers/formatio n professionnelle/53039 Le 23-7-2018

https://www.e-tribart.fr/blog/e-learning/difference-

formation-en-ligne-et-formation-a-distance

http://www.podcastfrancaisfacile.com17-9-2018

http://www.francailfacile.com11-8-2018

http://www.francaisinteractif7-6-2018

http://etudesslaves.edel.univ-

poitiers.fr/index.php?id=1207 26 septembre 2018.

https://www.auf.org/nouvelles/actualites/formation-

distance-pour-les-professeurs-de-fle/16-8-2018 
